\title{
Tunneling Between Multimode Stacked Quantum Wires
}

\author{
M. MACUCCI ${ }^{\mathrm{a}, *}$, A. T. GALICK ${ }^{\mathrm{b}}$ and U. RAVAIOLI ${ }^{\mathrm{b}}$ \\ ${ }^{a}$ Dipartimento di Ingegneria dell'Informazione, Via Diotisalvi, 2 I-56126 Pisa, Italy; \\ ${ }^{\mathrm{b}}$ Beckman Institute, $405 \mathrm{~N}$ Mathews Urbana, IL 61801
}

Tunneling between vertically stacked quantum wires has been investigated. The wires are assumed to have the dimension perpendicular to the tunneling barrier much smaller than the other transverse dimension, so that only the lowest mode in such direction is to be taken into account, while many modes in the other direction are filled. A model with hard-wall confinement has been used for the investigation of the transport problem, and the tunneling conductance has been computed, via a recursive Green's functions procedure.

Keywords: Quantum wire, tunneling, stacked 2DEGs, green's functions

\section{INTRODUCTION}

The possibility of fabricating heterostructures containing two weakly coupled and independently contacted 2DEGs (two-dimensional electron gases) has originated several proposals for possible devices exploiting controlled tunneling between such 2DEGs. The basic idea, proposed by Sakakibara et al. [1], consists in fabricating two stacked 2DEGs with different thicknesses, so that in normal conditions the ground states for the two quantum wells are not aligned. By applying a potential to a metallic gate obtained on top of the heterostructure, it is possible to alter the potential landscape and to line up the two ground states, so that tunneling will be possible. If we want to observe interference effects dependent on the length of the metallic gate, the situation is, however, more complex than what is discussed in Ref. [1]. While in a quantum wire the number of transverse modes is limited or we can even achieve monomode propagation, in a $2 \mathrm{DEG}$ the number of transverse modes is not limited and the continuous distribution of wavevectors may tend to wash out interference effects. In particular, the dependence of the tunneling conductance on the gate length will differ from that reported in Ref. [1] whenever the number of propagating modes is significantly larger than one.

This happens because each pair of modes contributing to conductance will be associated with a different transfer length, corresponding to

* Corresponding author. 
the length over which complete transfer from one waveguide to the other occurs. Therefore, as a function of the coupling length, the tunneling conductance will not exhibit a periodic behavior, but, rather, a superposition of different periodicities. In the limiting case of two laterally unconfined 2DEGs the number of occupied transverse subbands is practically infinite and any periodic pattern as a function of gate length should disappear. However, if the transverse dimension is limited by etching, thus defining two stacked quantum wires instead of two stacked 2DEGs, it is possible to limit the number of transverse modes contributing to conduction, and therefore to preserve some sort of periodic or quasi-periodic behavior. This is the case investigated in the present paper: we study the tunneling conductance between two quantum wires with rectangular cross-section (with a large aspect ratio, greater than 10) separated by a barrier along the longer side.

\section{MODEL}

Our model consists of two vertically stacked quantum wires with a rectangular cross section, as shown in Figure 1. At the outer ends, the two quantum wires are separated by hard walls and are therefore completely independent, so that the electron wave functions are completely localized

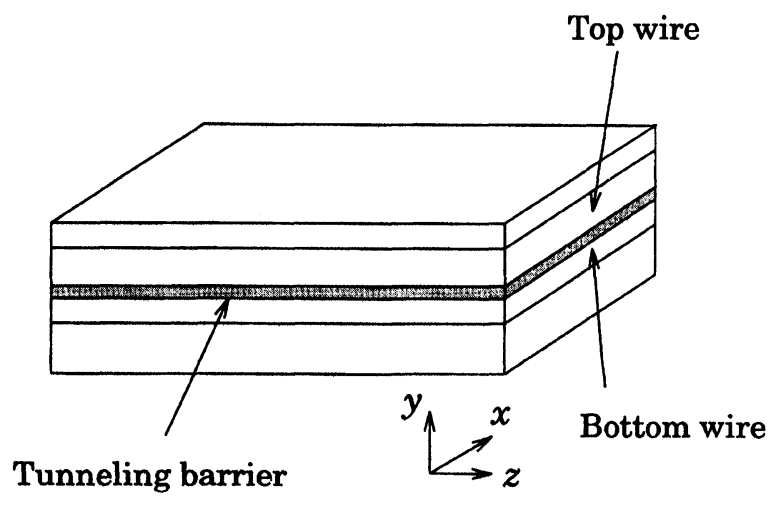

FIGURE 1 Sketch of the stacked quantum wire structure. in each of them, while in the "coupling region" they are separated by a tunneling barrier of finite height. The central section of the coupling region corresponds to the area covered by the metallic gate used to shift the potentials of the two quantum wires. This section is connected to the outer sections by means of a region with graded potentials, with a length corresponding to that over which the gate potential is effectively screened. In order to reduce the computer time used for the simulations and considering that most of the relevant physics is preserved unchanged, we have included only one intermediate section between the central region and the outer ends, with a potential landscape corresponding to an average of those relative to the adjacent sections. The gate bias is assumed to tilt the potential linearly along the vertical direction. In order to solve the transport problem along this structure, we use the quasi-3D approach that we developed for the investigation of electron waveguide couplers $[2,3]$ : the structure is divided, along the longitudinal direction, into a number of slices, each of which has a constant potential profile. The Schrödinger equation is solved within each slice and the eigenvalues and eigenfunctions thus obtained are used to evaluate the tunneling conductance by means of a recursive Green's function approach [2].

In the case of the present structure we are faced with an additional problem: the effective mass in the region corresponding to the barrier dividing the two quantum wells is different from that in the wells themselves. Let us assume a reference system with $y$ along the vertical direction, perpendicular to the tunneling barrier, $z$ along the longitudinal direction, parallel to current flow, and $x$ along the horizontal transverse direction (see Fig.1). The Schrödinger equation in each slice reads:

$$
\begin{aligned}
\frac{-\hbar^{2}}{2 m^{*}(y)} \frac{\partial^{2} \psi}{\partial x^{2}} & -\frac{\hbar^{2}}{2} \frac{\partial}{\partial y}\left[\frac{1}{m^{*}(y)} \frac{\partial \psi}{\partial y}\right] \\
& -\frac{\hbar^{2}}{2 m^{*}(y)} \frac{\partial^{2} \psi}{\partial z^{2}}+V(x, y) \psi=E \psi
\end{aligned}
$$


We can try a solution of the type $\psi=\chi(x, y)$ $\exp (i k z)$ (more generally it would be $\psi=\chi(x, y)$ $[A \exp (i k z)+B \exp (-i k z)]):$ the transverse equation (on the $x-y$ plane) can then be written as

$$
\begin{aligned}
\frac{-\hbar^{2}}{2 m^{*}(y)} \frac{\partial^{2} \chi(x, y)}{\partial x^{2}} & -\frac{\hbar^{2}}{2} \frac{\partial}{\partial y}\left[\frac{1}{m^{*}(y)} \frac{\partial \chi(x, y)}{\partial y}\right] \\
& -\frac{\hbar^{2}}{2 m^{*}(y)}\left(-k^{2}\right) \chi(x, y) \\
& +V(x, y) \chi(x, y)=E \chi(x, y) .
\end{aligned}
$$

If we define $\Delta m^{*}(y)=m^{*}(y)-m_{0}^{*}$, where $m_{0}^{*}$ is the effective mass in the quantum wells, and $E_{z}=\hbar^{2} /\left(2 m_{0}^{*}\right) k^{2}$, we get:

$$
\begin{aligned}
\frac{-\hbar^{2}}{2 m^{*}(y)} \frac{\partial^{2} \chi(x, y)}{\partial x^{2}} & -\frac{\hbar^{2}}{2} \frac{\partial}{\partial y}\left[\frac{1}{m^{*}(y)} \frac{\partial \chi(x, y)}{\partial y}\right] \\
& -E_{z} \frac{\Delta m^{*}(y)}{m_{0}^{*}+\Delta m^{*}(y)} \chi(x, y) \\
& +V(x, y) \chi(x, y)=\left(E-E_{z}\right) \chi(x, y) .
\end{aligned}
$$

We notice that if the term $E_{z} \Delta m^{*}(y) /\left(\left(m_{0}^{*}\right)\right.$ $\left.+\Delta m^{*}(y)\right)$ can be neglected compared to $V(x, y)$, this is just a standard 2D Schrödinger equation with variable effective mass. Such term is nonzero only within the barrier, where $\Delta m^{*}(y) /\left(m_{0}^{*}+\right.$ $\left.\Delta m^{*}(y)\right)$ is, however, always smaller than 0.5 (0.44 for GaInAs/AlInAs and 0.264 for GaAs/ $\mathrm{AlGaAs})$. Furthermore it is clearly $E_{z}<E_{\mathrm{f}}$, where $E_{\mathrm{f}}$ is the Fermi level. Thus, as long as we choose values of the Fermi level that are less than one half of the barrier height, the transverse problem can be reduced to that of solving a conventional 2D Schrödinger equation, without introducing too large an error.

\section{NUMERICAL PROCEDURE AND RESULTS}

The Schrödinger equation in each transverse slice is discretized on a uniform finite-difference mesh, and the eigenvalue problem is solved with an efficient iterative Chebishev-Arnoldi technique.
The typical size of the mesh varies between $20 \times$ 50 and $20 \times 350$ points, and the lowest eigenvalues up to the 50th are evaluated. The presence of a varying effective mass along the $y$ direction is treated with the standard formalism ensuring the continuity of the wave function and of its normal derivative at the interface [4], as shown in Eq. (1). The transmission coefficients between the two waveguides are then computed with the modified recursive Green's function method [3], and finally averaged over the derivative of the Fermi function, in order to take into account the effects of temperature [5] (all of our calculations have been performed for a temperature of $1.6 \mathrm{~K}$ ).

We have taken into consideration the parameter values for two different material systems: a) GaInAs/AlInAs and b) GaAs/AlGaAs. For material system a) we have considered an effective mass $m^{*}=0.042 m_{0}$ in GaInAs and $m^{*}+\Delta m^{*}=0.075$ $m_{0}$ in the barrier region, $m_{0}$ being the mass of the free electron. A value of $0.5 \mathrm{eV}$ has been chosen for the conduction band discontinuity. For material system b) an effective mass $m^{*}=0.067 m_{0}$ has been considered in GaAs and $m^{*}+\Delta m^{*}=0.091 m_{0}$ in the barrier region. The conduction band discontinuity has been assumed to be $0.3 \mathrm{eV}$.

In all the cases we have studied, the thicknesses of the heterostructure layers have been kept constant, while the width along $x$ of the quantum wires has been varied. The thickness of the upper wire along $y$ is $9 \mathrm{~nm}$, that of the lower wire $7 \mathrm{~nm}$ and that of the barrier $4 \mathrm{~nm}$. All the energies are indicated with reference to that of the lowest edge of the bottom quantum wire.

In Figure 2 we report the tunneling conductance between the input of one wire and the output of the other, in units of the conductance quantum $G_{0}=2 e^{2} / h$, for a GaInAs/AlInAs structure with widths of $50 \mathrm{~nm}$ (dotted line), $100 \mathrm{~nm}$ (dashed line) and $200 \mathrm{~nm}$ (solid line). The tunneling conductance is plotted versus the potential shift applied to the upper edge of the top quantum wire (the potential, as discussed before, is assumed to vary linearly in the vertical direction). We have considered only positive values of such shift, since 


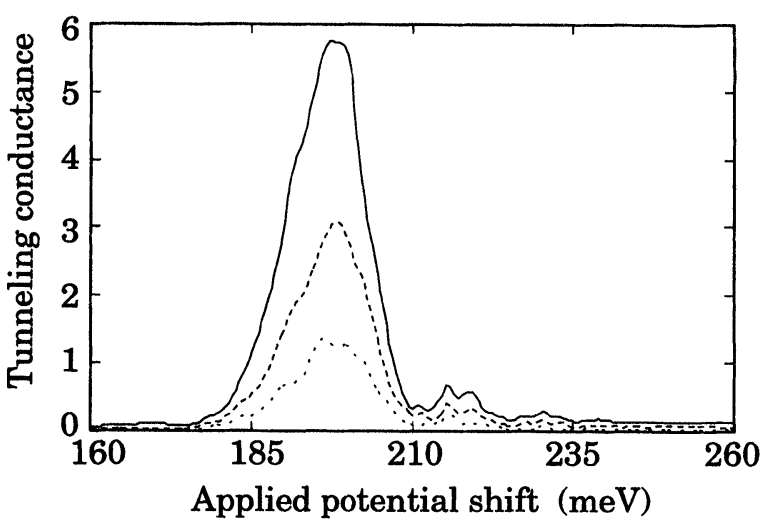

FIGURE 2 Tunneling conductance (in units of the conductance quantum) for the GaInAs/AllnAs structure versus the applied potential shift, with wire widths of $50 \mathrm{~nm}$ (dotted line), $100 \mathrm{~nm}$ (dashed line) and $200 \mathrm{~nm}$ (solid line). The Fermi level has been set at $0.24 \mathrm{eV}$.

the top wire is thicker than the bottom one and thus it needs to be raised to a higher potential in order to line up the energy levels in the two wires. The coupling length is $200 \mathrm{~nm}$.

For the results shown in this figure, a Fermi level of $0.24 \mathrm{eV}$ has been considered and up to 50 transverse modes have been included in the calculation. A clear peak, reaching a maximum for a bias of approximately $198 \mathrm{meV}$, is visible, corresponding to the line-up of the energy levels associated with the subbands in the two wires. At this point it is useful to draw a comparison between the situation for devices in which the coupled wires are obtained by lateral confinement [6] in the same 2DEG and the present situation. In the former case all the subbands line up for the same bias condition only if the two wires are identical and, even in such a case, only the highest ones give a significant contribution to the tunneling current, because they have the largest transverse wave vectors, in the direction orthogonal to the potential barrier. In the latter case, instead, the spacing between the subbands is determined by the width of the wire along $x$, which is the same for both wires, and therefore all of them line up at the same time. More importantly, all the modes give a similar contribution to the tunneling current, because the wave vector orthogonal to the tunneling barrier is the same for all of them. The result is that if the width of the wire increases, the height of the conductance peak also increases, due to the contribution of a larger number of modes, but its shape or position does not vary significantly.

In Figure 3 we report the tunneling conductance results for the GaAs/AlGaAs material systems, for structures $50 \mathrm{~nm}$ (thin line) and $100 \mathrm{~nm}$ (thick line) wide. Also in this case the coupling length (corresponding to the length of the biasing gate) is $200 \mathrm{~nm}$, while the Fermi level has been set at $0.15 \mathrm{eV}$. The peak is located approximately at $95 \mathrm{meV}$ : a value much lower than that in Figure 2, because of the significant difference in the effective mass value.

We have then investigated the behavior of the tunneling conductance as a function of the coupling length. If only one mode were contributing to the tunneling current, we would expect the sinusoidal oscillation predicted also by the coupled mode theory used for the analysis of optical waveguides; in this case, however, there are many contributing modes, each of which is associated with a different transfer length. As a result, the tunneling conductance exhibits some complex structure, depending on the number of modes

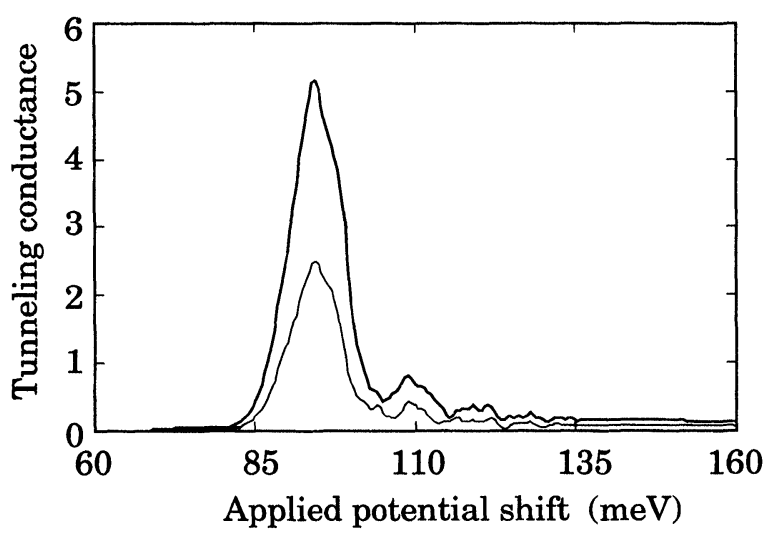

FIGURE 3 Tunneling conductance (in units of the conductance quantum) for the GaAs/AlGaAs structure versus the applied potential shift, with wire widths of $50 \mathrm{~nm}$ (thin line) and $100 \mathrm{~nm}$ (thick line). The Fermi level has been set at $0.15 \mathrm{eV}$. 
and on the relationships between the related transfer lengths.

In Figure 4 the tunneling conductance is plotted versus the length of the coupling region, for the GaInAs/AlInAs material system and for three different choices of the parameters. Two curves have been obtained for a width of $50 \mathrm{~nm}$ and a Fermi level of $0.22 \mathrm{eV}$ (dashed line) and of $0.24 \mathrm{eV}$ (thin line). The third curve (thick line) is for a width of $200 \mathrm{~nm}$ and a Fermi level of $0.24 \mathrm{eV}$. In all three cases the applied potential shift is $196 \mathrm{meV}$. A rather complex structure can be observed, due to the simultaneous presence of several pairs of coupled modes, each of which is characterized by a different transfer length. For the lowest value of the Fermi level we have an almost monomode propagation and the tunneling conductance oscillates sinusoidally.

Results for the GaAs/AlGaAs material system are reported in Figure 5, where the thick curve and the thin curve have been obtained for a Fermi level of $0.16 \mathrm{eV}$ and wire widths of $200 \mathrm{~nm}$ and of $50 \mathrm{~nm}$, respectively. The dashed curve is instead for a wire width of $50 \mathrm{~nm}$ and a Fermi level of $0.13 \mathrm{eV}$. The applied potential shift is $100.5 \mathrm{meV}$. Also for this material system we observe a transition from a

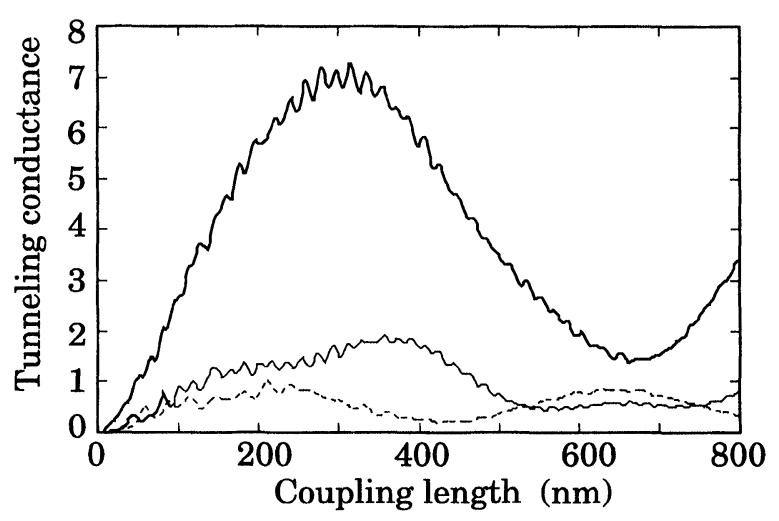

FIGURE 4 Tunneling conductance (in units of the conductance quantum) for the GaInAs/AlInAs structure versus the coupling length, with a wire width of $50 \mathrm{~nm}$ and a Fermi level set at $0.24 \mathrm{eV}$ (thin line) or at $0.22 \mathrm{eV}$ (dashed line). The thick line represents the results for a wire width of $200 \mathrm{~nm}$ and a Fermi level set at $0.24 \mathrm{eV}$. For all three cases the applied bias potential is $196 \mathrm{meV}$.

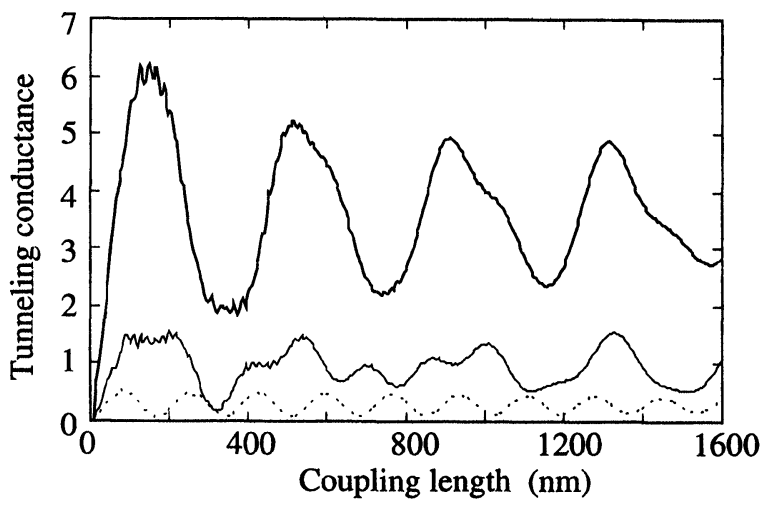

FIGURE 5 Tunneling conductance (in units of the conductance quantum) for the $\mathrm{GaAs} / \mathrm{AlGaAs}$ structure versus the coupling length, with a wire width of $50 \mathrm{~nm}$ and a Fermi level set at $0.16 \mathrm{eV}$ (thin line) or at $0.13 \mathrm{eV}$ (dashed line). The thick line represents the results for a wire width of $200 \mathrm{~nm}$ and a Fermi level set at $0.16 \mathrm{eV}$. For all three cases the applied bias potential is $100.5 \mathrm{meV}$.

quasisinusoidal dependence of the tunneling current on the coupling length (when just one pair of modes contributes) to a rather complex behavior.

\section{CONCLUSIONS}

We have investigated the tunneling conductance between two stacked quantum wires, as a function of an applied transverse electric field and of the coupling length. The conductance peak that is observed when the subbands in one wire line up with those in the other wire has a height depending on the total number of occupied subbands. Thus a relatively low "on" resistance can be achieved, which makes the investigated structure suitable for operation as a controlled switch.

\section{Acknowledgement}

This work has received partial support from the NATO Collaborative Research Grant n. 950753 and NSF grant ECS 95-26127. One of the authors (M. M.) acknowledges useful discussion with Michele Governale, Freek Prins and Heiko Fresser, and support from the Italian Ministry of the University and Scientific Research. 


\section{References}

[1] Sakakibara, H., Noguchi, M., Thornton, T. J., Hirakawa, $\mathrm{K}$. and Ikoma, T., "Electron Wave Switch with Distributed Coherent Resonant Tunneling Coupling", in the Proceedings of the International Electron Device Meeting IEDM 93-411.

[2] Macucci, M., Ravaioli, U. and Kerkhoven, T. (1992). "Analysis of Electron Transfer between Parallel Quantum Wires", Superlattices and Microstructures, 12, 509.

[3] Macucci, M., Galick, A. and Ravaioli, U. (1995). "Quasithree-dimensional Green's-function simulation of coupled electron waveguides", Phys. Rev. B, 52, 5210.

[4] Ando, Y. and Itoh, T. (1987). "Calculation of transmission tunneling currents across arbitrary potential barriers", Journal of Appl. Phys., 61, 1497.

[5] Wees, J., van, Kouwenhoven, L. P., Willems, E. M. M., Harmans, C. J. P. M., Mooji, J. E., Houten, H. van, Beenakker, C. W. J., Williamson, J. G. and Foxon, C. T. (1991). "Quantum ballistic and adiabatic electron transport studied with quantum point contacts", Phys. Rev. B, 43, 12431.

[6] del Alamo, J. A. and Eugster, C. C. (1990). "Quantum field-effect directional coupler”, Appl. Phys. Lett., 56, 78.

\section{Authors' Biographies}

Massimo Macucci received the Laurea in Electronic Engineering in 1987 from the University of Pisa, Italy, and the Ph.D. in Electrical Engineering from the University of Illinois, in 1993. He is currently serving on the faculty of the Electrical Engineering Department at the University of Pisa, Italy. His research interests include quantuminterference and single-electron devices, Coulomb Blockade phenomena, modeling and measurements of noise in electron devices.

Albert Galick received the B.S. degree in mathematics from the Massachusetts Institute of Technology in 1980, the M.S. degree in Mathematics in 1984 and the Ph.D. Degree in Computer Science in 1993. He worked as programmer with AT\&T in 1980 and from 1984 to 1986. Since 1993 he is a post-doctoral research associate at the Beckman Institute of the University of Illinois.

Umberto Ravaioli received the Laurea in Electronic Engineering in 1980 and the Laurea in Physics in 1982, from the University of Bologna, Italy, and the Ph.D. in Electrical Engineering from Arizona State University, in 1986. He is now a Professor of Electrical and Computer Engineering at the University of Illinois, UrbanaChampaign. 

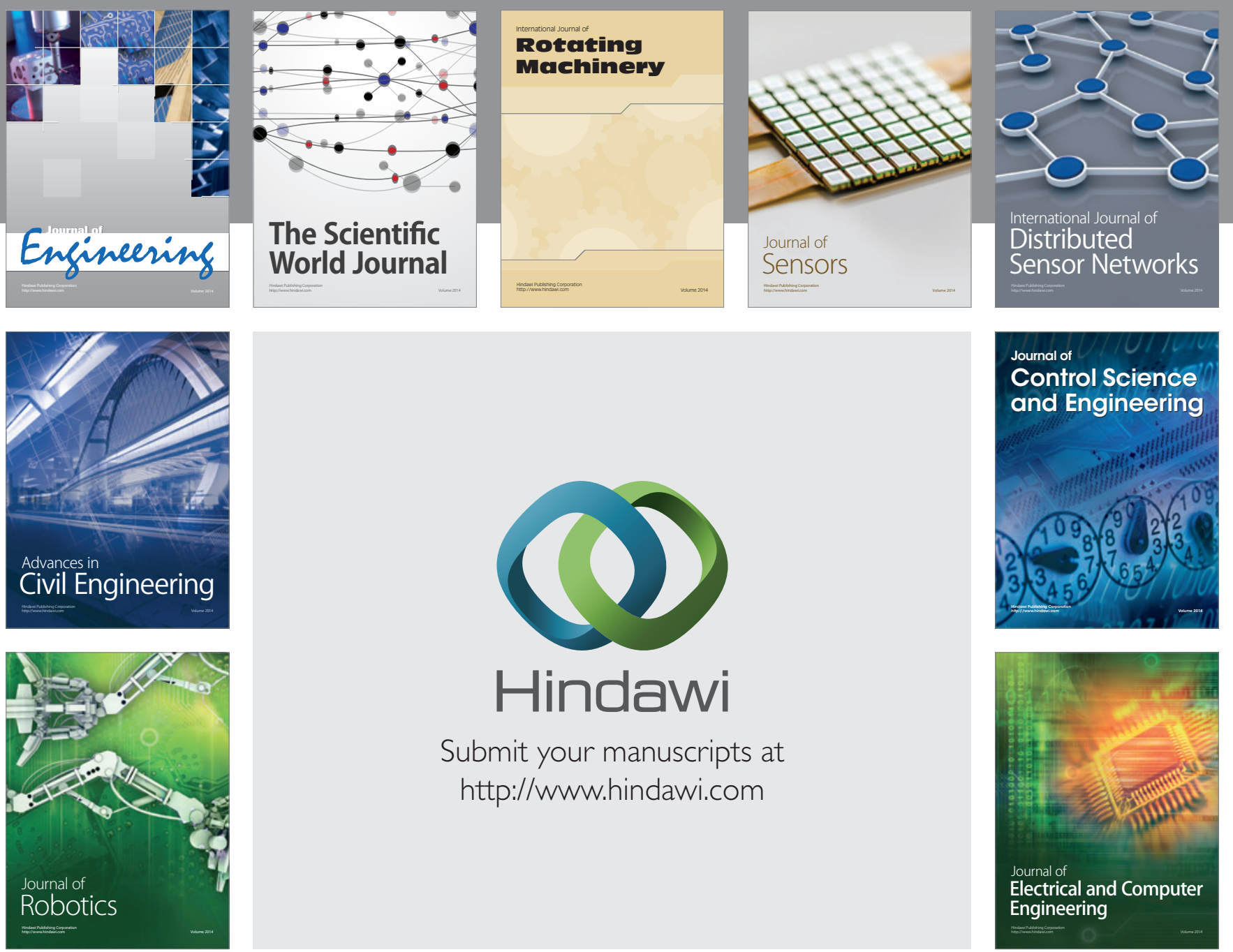

Submit your manuscripts at

http://www.hindawi.com
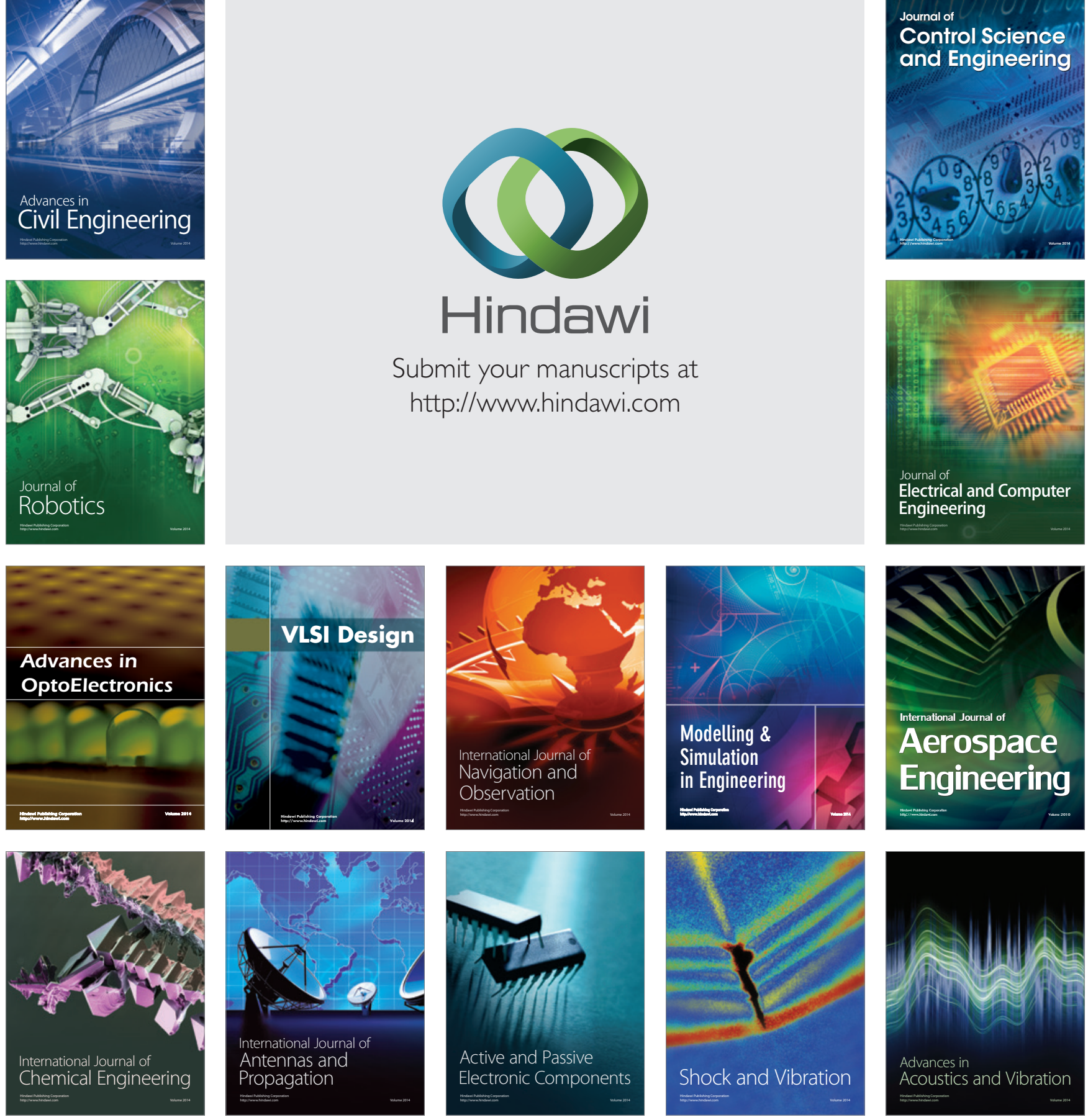